\title{
Dislocations in GaAs p-i-n diodes grown by hydride vapour phase epitaxy
}

\author{
A. Säynätjoki · A. Lankinen · T. O. Tuomi · \\ P. J. McNally · A. Danilewsky $\cdot$ Y. Zhilyaev · \\ L. Fedorov
}

(C) Springer Science+Business Media, LLC 2007

\begin{abstract}
Hydride vapour phase epitaxy grown allepitaxial p-i-n structures were studied by synchrotron $\mathrm{X}$-ray topography. Three types of process induced dislocations were found: short threading dislocations, long straight interfacial dislocations and circular arc dislocations. The majority of the dislocations observed are short straight threading dislocations, the density of which is typically about $5000 \mathrm{~cm}^{-2}$. The dislocations at the $\mathrm{p}-\mathrm{i}$ interface are long straight lines parallel to [110]. They are screw dislocations having their Burgers vector parallel to [110], calculated from the contrast analysis of the well resolved dislocation images. One sample also showed a dense misfit dislocation network at the n-side. However, no misfit dislocations were seen in the back-reflection topographs of the $\mathrm{n}$-side of the other samples, which shows that it is possible to grow a misfit-dislocation free n-type GaAs layer onto the substrate side of a hydride vapour phase epitaxy grown GaAs surface after proper substrate removal.
\end{abstract}

A. Säynätjoki $(\varangle) \cdot$ A. Lankinen · T. O. Tuomi

Micro and Nanosciences Laboratory, Helsinki University of

Technology, Micronova, Espoo, Finland

e-mail: antti.saynatjoki@tkk.fi

P. J. McNally

RINCE, Dublin City University, Dublin, Ireland

A. Danilewsky

Kristallographisches Institut, Universität Freiburg, Freiburg, Germany

Y. Zhilyaev · L. Fedorov

A. F. Ioffe Physico-Technical Institute, St. Petersburg, Russia

\section{Introduction}

Hydride vapour phase epitaxy (HVPE) is a rapid growth technique, which is suitable for depositing thick ultra-pure as well as doped epitaxial layers on top of a substrate wafer [1]. Thick HVPE grown GaAs layers have been used in various types of devices, such as lasers [2] and nonlinear optics devices [3]. Thick layers also enable free-standing epitaxial structures, which are used e.g. as GaN substrates $[4,5]$. Ultra-pure all-epitaxial materials are also desired in detector manufacturing. Such GaAs p-i-n diode structures, grown by open tube chloride HVPE, are studied in this work by synchrotron $\mathrm{X}$-ray topography. The main interest in this work is the defect structure of the samples. In detectors one of the important properties is the leakage current, upon which defects like dislocations are thought to have an influence. The dislocations may attract harmful residual impurities, and in this way act as gettering centres. Contrary to such a beneficial effect the dislocations may actually behave as channels for electric current through the diode and worsen its device performance. In either case it is important to know the defect structure of the HVPE grown p-i-n diode. X-ray diffraction topography using synchrotron radiation is an ideal detection method for measuring the dislocation density as well as determining the type and spatial distribution of the dislocations [6]. The technique is also rapid compared to those using conventional X-ray sources. It can be applied in different measuring geometries both in the transmission and in the back-reflection modes without any careful alignment of the sample.

\section{Experimental}

The HVPE process utilizes $\mathrm{Ga}$ metal and $\mathrm{AsCl}_{3}$ as the source materials with $\mathrm{H}_{2}$ as the carrier gas. $\mathrm{H}_{2}$ reacts with 
$\mathrm{AsCl}_{3}$ to form $\mathrm{HCl}$ and $\mathrm{As}_{4}$. The $\mathrm{HCl}$ reacts with $\mathrm{Ga}$ to form $\mathrm{GaCl}$, which reacts with the $\mathrm{As}_{4}$ to form $\mathrm{GaAs}$ on the substrate.

The GaAs is transported to the substrate where it forms an epitaxial layer. The VPE installation comprises a gas unit for controlling the flow of gases, a quartz reactor and a furnace for maintaining the temperature regime in the reactor. The major chemical reactions between the starting components $\mathrm{Ga}, \mathrm{AsCl}_{3}$ and $\mathrm{H}_{2}$ are as follows:

$\mathrm{AsCl}_{3}+\frac{3}{2} \mathrm{H}_{2} \leftrightarrow \frac{1}{4} \mathrm{As}_{4}+3 \mathrm{HCl}$

$\mathrm{HCl}+\mathrm{GaAs} \leftrightarrow \mathrm{GaCl}+\frac{1}{4} \mathrm{As}_{4}+\frac{1}{2} \mathrm{H}_{2}$

The reaction $(1,2)$ takes place in the source zone of the chamber running mainly from left to right. The reaction (1) takes place in the gaseous phase (homogeneous reaction). A gallium arsenide layer is formed on the liquid gallium surface due to the presence of arsenic in the gas phase. Products of the reaction $(1,2)$ flow into the growth zone, where reaction (2) changes direction from right to left at lower temperatures. In these circumstances, gallium arsenide epilayers are deposited on the substrates due to heterogeneous reactions. The GaAs growth rate and crystal quality are a complicated function of temperature, oversaturation of active components, gas flow rate and also of the system geometry and gallium source surface area. The process is highly selective and acts to form high-purity GaAs. By operating the growth system continuously at a relatively slow growth rate, the purity of the growing material can be controlled to a high degree. The process described above was utilized to grow samples studied in this work. The samples studied in this work were grown by HVPE on silicon doped vertical gradient freeze (VGF) grown $\mathrm{n}^{+}$-GaAs substrates $\left(\mathrm{n}=2 \times 10^{18} \mathrm{~cm}^{-3}\right)$. The surface orientation was (100) with a $4^{\circ}$ miscut angle toward
$<111>$. First, an undoped intrinsic 100-200 $\mu$ m-thick GaAs layer was grown at $700{ }^{\circ} \mathrm{C}$. Subsequently, a $5 \mu \mathrm{m}$ thick zinc doped p-type layer was grown in the same HVPE reactor on top of the i-layer. The hole concentration of the p-type layer was as large as $10^{18} \mathrm{~cm}^{-3}$. In order to make a thin n-type layer on the other side of the sample, the substrate was ground and etched away. After the substrate had been removed a $2-3 \mu$ m-thick n-type layer doped with tin was grown by HVPE on the i-side of the sample. In this way, a number of $\mathrm{p}$-i-n diode structures were obtained for further investigations, for which synchrotron X-ray topography studies of three samples, denoted as D85, D87 and D89, are reported in this work.

The synchrotron X-ray topographs were made at the HASYLAB-DESY F1 topography measuring station using a continuous spectrum of radiation from a bending magnet source, and Slavich VRP-M high-resolution films. Largearea transmission, section transmission, large-area backreflection and grazing-incidence reflection measurement geometries were employed to record a number of Laue patterns of high-resolution diffraction topographs, which were magnified with a microscope.

\section{Results and discussion}

\subsection{Dislocation structure}

Figure 1 shows 331 and 602 large-area transmission topographs of sample D89 having an approx. 100-200 $\mu \mathrm{m}$ thick i-layer and a $5 \mu \mathrm{m}$-thick p-layer. The two topographs in Fig. 1 are a stereo pair, from which a three-dimensional image can be viewed e.g. with crossed eyes by looking at the left and the right image with the right and the left eye, respectively. There are three distinct types of dislocations visible in Fig. 1: short threading dislocations, circular arc dislocations and long straight dislocations.
Fig. 1 Stereo pair 331 of and 602 transmission topographs of sample D89
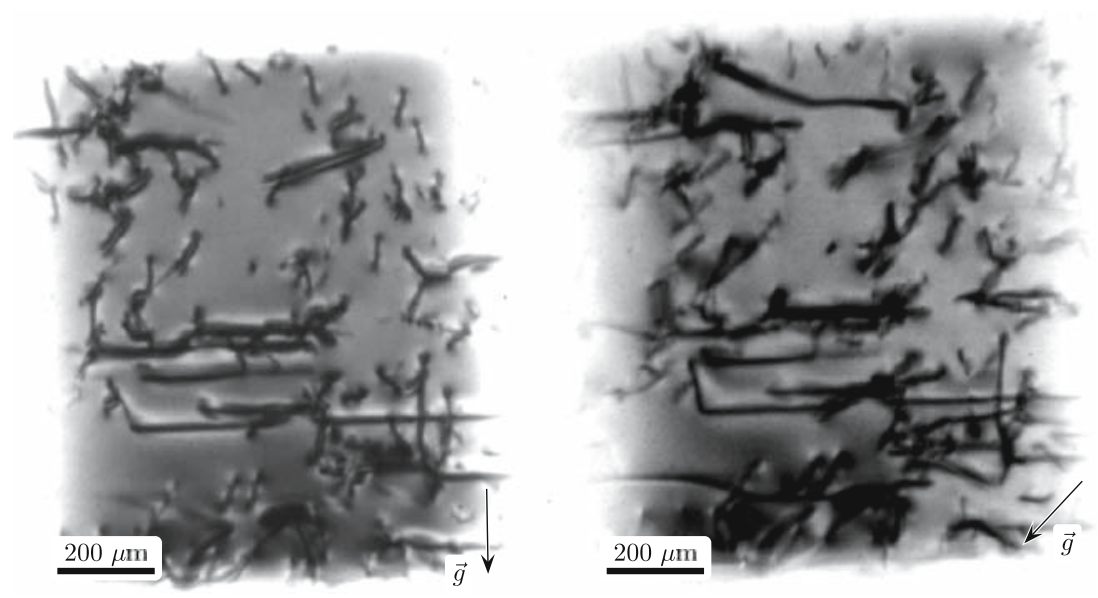
The majority of the dislocations are threading dislocations, which penetrate the sample as seen from the stereo image of Fig. 1. The circular arc dislocations are similar to those observed in the undoped GaAs wafers grown by both VGF [7] and vapour pressure controlled Czochralski (VCz) [8] methods, but the density of these dislocations is significantly smaller in the HVPE samples studied in this work. The threading dislocations are clearly seen in the section transmission topograph of the same sample (Fig. 2). The grey thick horizontal lines in the topograph are likely to be images of the $\mathrm{p}$ - and n- layers. They might also be Pendellösung fringes explained by the dynamical theory. However, Pendellösung fringes are rarely seen in GaAs topographs and the existence of fringes is unlikely in this sample with a relatively large number of dislocations.

The long dislocations parallel to [110] are found to be at the p-i interface by using back-reflection topography. The $1 \overline{1} 510^{\circ}$ - grazing-incidence back-reflection topograph of Fig. 3 shows the dislocations at the p-i interface. Due to geometry of the grazing-incidence measurement, the vertical dimension in Fig. 3 is contracted by fraction $\left(1 / \tan 10^{\circ}\right)$ Figure 3 also shows the ends of the threading dislocations very clearly. Their density is about $5,000 \mathrm{~cm}^{-2}$.

Figures 4 and 5a show 206 back-reflection topographs of $\mathrm{p}$ - and n-side of sample D87, respectively. As a rule, the interfacial dislocations are observed in the p-side topograph (Fig. 4) in two mutually perpendicular <110> directions. The n-side topograph of Fig. 5a) does not show interfacial dislocations, which confirms that the interfacial dislocations reside near the $\mathrm{p}-\mathrm{i}$ interface of the sample. However, for sample D85, the n-side back-reflection topographs show a dense misfit dislocation network with a dislocation density of about $20 / \mathrm{mm}$ (Fig. 6a), which is significantly larger than the density observed in the $\mathrm{p}-\mathrm{i}$ interface of all the samples.

Figures $5 \mathrm{~b}$ and $6 \mathrm{~b}$ show three-dimensional schematic drawings of the dislocation structure related to the interfacial dislocations in the samples. Some of the threading dislocations in the intrinsic layer continue into the $\mathrm{p}-\mathrm{i}$ interface as straight dislocations in the $<110>$ directions. The length of the interfacial dislocations is usually a few hundred micrometers. The straight dislocations turn into threading dislocations in the p-layer. The n-layer is usually free of interfacial dislocations, as seen from Fig. 5a. Figure 6a shows a misfit dislocation network, which is explained by the incomplete removal of the substrate. The schematic drawing of Fig. 6b suggests that the misfit dislocation network is created at the $\mathrm{i}$-substrate interface and

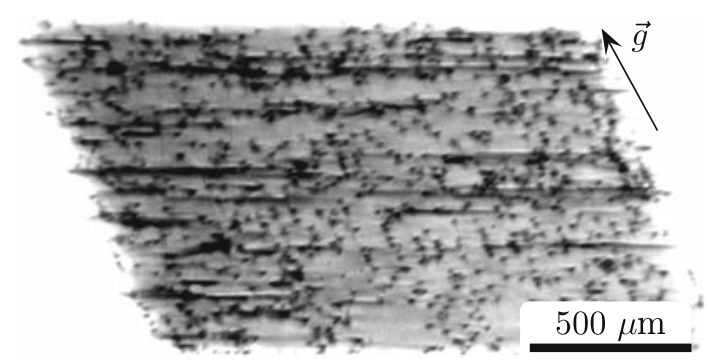

Fig. $31 \overline{1} 510^{\circ}$ - grazing-incidence reflection topograph of p-side of sample D89

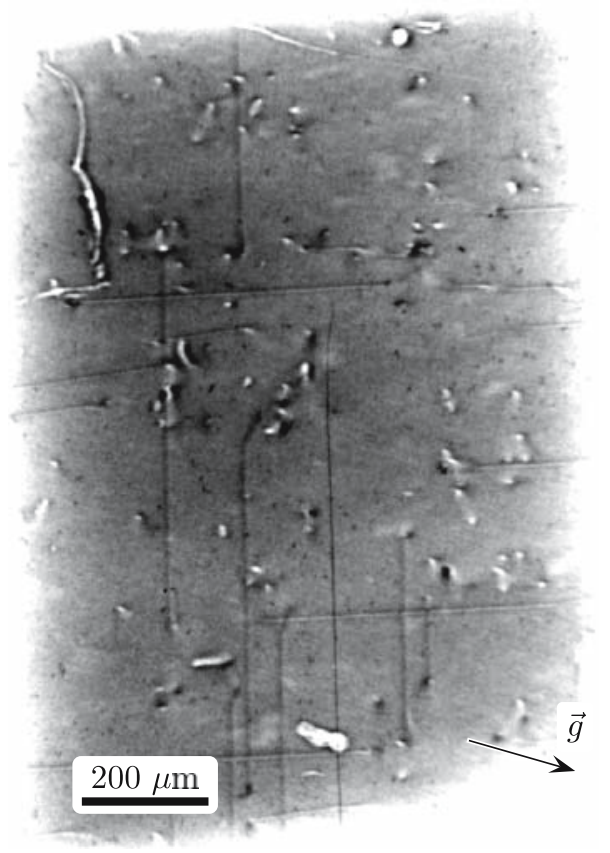

Fig. 4206 back reflection topograph of p-side of sample D87

it is left there because of the remains of the substrate in sample D85. The thicknesses in Figs. 5b and $6 \mathrm{~b}$ are not in scale in order to better illustrate the structure.

\subsection{Lattice mismatch}

Doping differences between different layers cause lattice mismatch, because the size of the doping atom is different from that of the corresponding atom in the undoped lattice. As a simple model, the nearly intrinsic epitaxial layer is assumed to be pure GaAs, and mismatch is estimated to arise from the difference of the volume occupied by the doping atom compared to the atom that it substitutes. The

Fig. 2462 section transmission topograph of sample D89

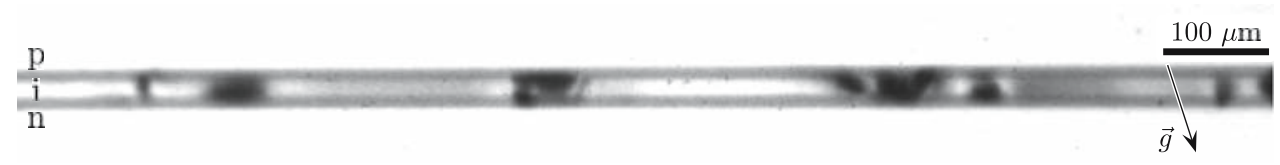


Fig. 5 (a) 206 back reflection topograph of n-side of sample D87, (b) schematic drawing of interfacial dislocations and related threading dislocations

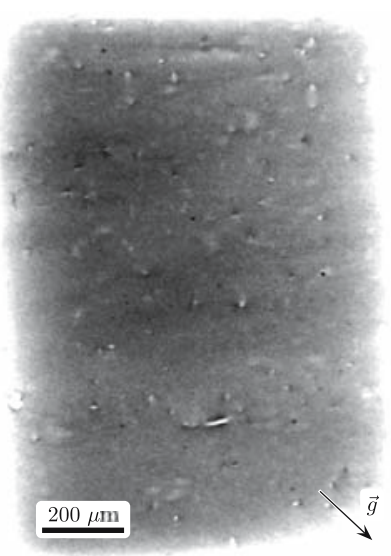

(a)

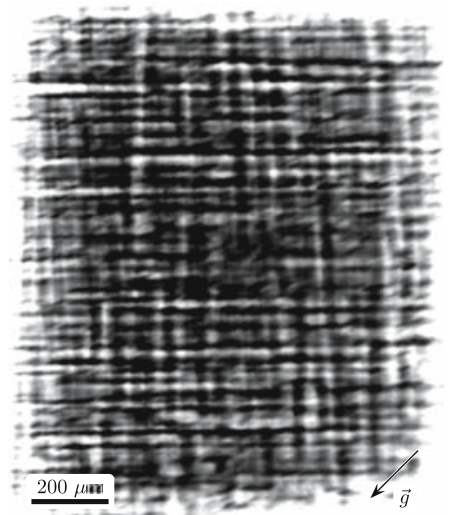

(a)

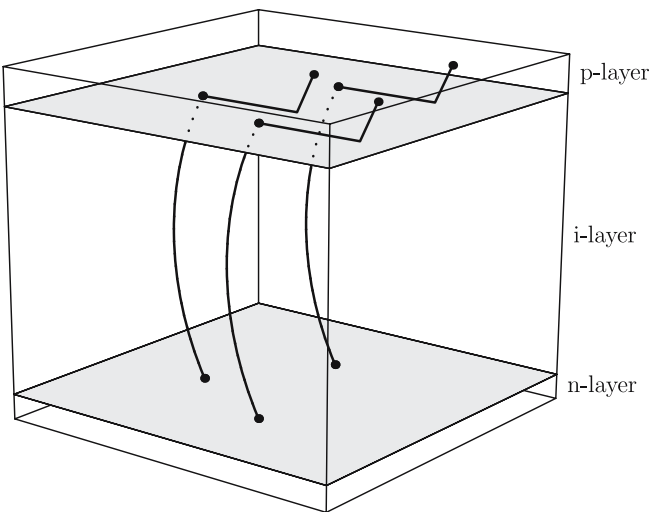

(b)
Fig. 6 (a) 206 back reflection topograph of n-side of sample D85, (b) schematic drawing of interfacial dislocations and threading dislocations related to p-side interfacial dislocations

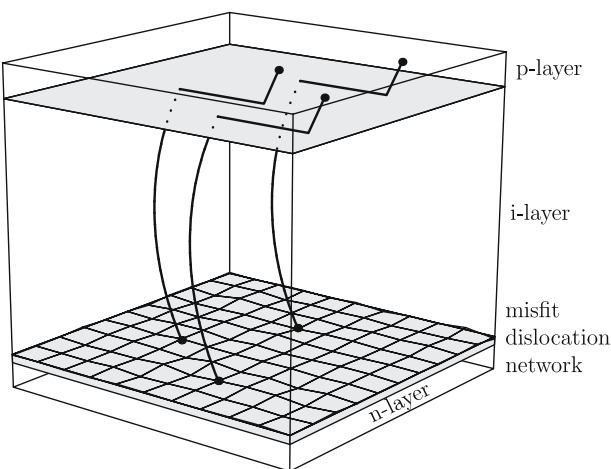

(b) p-doped layer contains $10^{18} \mathrm{Zn}$ atoms per $\mathrm{cm}^{3}$ and each $\mathrm{Zn}$ atom is assumed to substitute a $\mathrm{Ga}$ atom. In the following we compare between the volumes of a $\mathrm{Ga}$ and $\mathrm{Zn}$ atom surrounded by As atoms. One must also take into account that the doping atoms are ionized, which may change their size significantly. Therefore the calculations were made using the sizes of $\mathrm{Ga}^{3+}, \mathrm{As}^{3-}$ and $\mathrm{Zn}^{2+}$ ions [9]. This is not the exact state of the atoms in the GaAs lattice, where the bonds are covalent-like, but it gives an estimate for the size occupied by an atom and its bonds to its neighbours in the lattice. The "radius" of the gallium atom in the GaAs lattice is estimated to be $r_{\mathrm{Ga}: \mathrm{GaAs}}=\frac{1}{2}\left(r_{\mathrm{Ga}^{3+}}+r_{\mathrm{As}^{3-}}\right)=$ $\frac{1}{2}(0.65 \AA+1.91 \AA)=1.28 \AA$, and the same for the $\mathrm{Zn}$ atom that substitutes a $\mathrm{Ga}$ atom in the GaAs lattice, and is ionized for a p dopant, $r_{\mathrm{Zn}: \mathrm{GaAs}}=\frac{1}{2}\left(r_{\mathrm{Zn}^{2+}}+r_{\mathrm{As}^{3-}}\right)=$ $\frac{1}{2}(0.83 \AA+1.91 \AA)=1.37 \AA$. Therefore, the volume difference per doping atom is

$$
\begin{aligned}
\Delta V_{\mathrm{Zn}, \mathrm{Ga}: \mathrm{GaAs}} & =\frac{4 \pi}{3}\left(r_{\mathrm{Zn}: \mathrm{GaAs}}^{3}-r_{\mathrm{Ga}: \mathrm{GaAs}}^{3}\right) \\
& =\frac{4 \pi}{3}\left[(1.37 \AA)^{3}-(1.28 \AA)^{3}\right]=1.99 \AA^{3} .
\end{aligned}
$$

With a doping concentration of $10^{18} \mathrm{~cm}^{-3}$ this makes a relative lattice volume mismatch $\frac{\Delta V}{V}=10^{18} \mathrm{~cm}^{-3} \times$ $1.99 \AA^{3}=1.99 \times 10^{-6}$. The small relative mismatch of unit cell dimension $\frac{\Delta l}{l}$ can be calculated from the volume difference $\frac{\Delta V}{V}$ :

$\frac{\Delta l}{l}=\frac{1}{3} \frac{\Delta V}{V}=\frac{1}{3} \times 1.99 \times 10^{-6}=6.62 \times 10^{-7}$.

If all the stress due to lattice mismatch is assumed to be relaxed into a misfit dislocation network, distance between two adjacent dislocations is expected to be

$\frac{a_{\mathrm{GaAs}}}{\left|\frac{\Delta l}{l}\right|}=\frac{5.6533 \times 10^{-7} \mathrm{~mm}}{6.62 \times 10^{-7}}=0.85 \mathrm{~mm}$.

This corresponds to about one dislocation per $\mathrm{mm}$, which is in a good agreement with the topographs of Figs. 1, 3, 4 and 5 a), especially if one takes into account the simplifications used in the calculation of the lattice mismatch.

The size of the Si doping atom can be estimated to be $r_{\mathrm{Si}: G a A s}=\frac{1}{2}\left(r_{\mathrm{Si}^{4+}}+r_{\mathrm{As}^{3-}}\right)=\frac{1}{2}(0.40 \AA+1.91 \AA)=1.155 \AA$ 
[9]. A calculation similar to that in Eqs. (3) through (5) gives a lattice mismatch of $-1.55 \times 10^{-6}$ and a misfit dislocation density of about $3 / \mathrm{mm}$ for the interface between the $\mathrm{n}^{+}$-doped substrate and i-layer. This is somewhat smaller than the density of the misfit dislocation network seen in the topograph of Fig. 6a, but the calculations were made using electron concentration instead of the actual $\mathrm{Si}$ atom concentration. However, comparison between Hall measurements and SIMS measurements of VGF grown $\mathrm{Si}$ doped GaAs [10] show that less than $50 \%$ of the Si atoms contribute as $\mathrm{n}$ dopants. Therefore, the dense misfit dislocation network in the n-side of sample D85 is believed to get rise from this lattice mismatch.

The epitaxial n-layer is doped with $10^{17} \mathrm{~cm}^{-3} \mathrm{Sn}$ atoms $\left(r_{\mathrm{Sn}^{4+}}=r_{\mathrm{Ga}^{3+}}=0.65 \AA\right.$ [9]), and our simple estimate gives an exact lattice match to intrinsic GaAs. Indeed, the n-side topographs of sample D87 (Fig. 5a) shows no misfit dislocations. Only the ends of the threading dislocations are seen. This shows that it is possible to grow a misfit dislocation free n-layer onto the i-layer after a successful substrate removal process.

The topographs of sample D85 show that misfit dislocations may arise at the interface of the n-doped substrate and the epitaxial i-layer (Fig. 6a). However, all the samples were grown on similar substrates, but not all of them show these dislocations (Fig. 5a). The difference is believed to be in the substrate removal process. The exact end point of the thinning process is relatively difficult to control. Thus it is possible that only for some samples has the dislocation network been fully removed, resulting in a misfit dislocation free surface for subsequent growth. In any case, threading dislocations remain in the i-layer and are the starting points of the misfit dislocations at the $\mathrm{p}-\mathrm{i}$ interface, as seen in the stereo pair of Fig. 1. Using undoped rather than n-doped substrates would improve lattice match between the substrate and the thick i-layer. This could reduce threading dislocation density in the i-layer, which might also reduce the number of dislocations in the p-side of the diode.

\subsection{Burgers vector analysis}

The image of a dislocation disappears in a diffraction topograph when $\vec{g} \cdot \vec{b}=0$, where $\vec{g}$ is the diffraction vector and $\vec{b}$ is the Burgers vector of the dislocation [11]. Figure 7 shows the 004, $\overline{1} 17$ and $1 \overline{1} 7$ back-reflection topographs of the p-side of sample D87 from the same film as the 206 topograph of Fig. 4. Figure 4 shows images of straight dislocations along both [110] and [1ㅜㅣ] directions. They all disappear with the 004 reflection shown in Fig. 7a. The $\overline{1} 17$ and $1 \overline{1} 7$ topographs in Figs. $7 \mathrm{~b}$ and $7 \mathrm{c}$ show straight dislocations only along the [1시 direction. From the disappearance of the straight dislocations along the [110] direction in all these topographs it is concluded that they are screw dislocations having their Burgers vector parallel to [110]. The discovery of the interfacial screw dislocations is rather unexpected, but is in a good agreement with theoretical results on misfit dislocation formation [12]. Furthermore, pure screw misfit dislocations have been previously found in $\mathrm{ZnSe}$ films grown on GaAs [13].

\section{Conclusions}

A characteristic feature of the HVPE grown intrinsic GaAs is the rather large density of threading dislocations, the origin of which is believed to be the misfit dislocation network between the n-type substrate and the epitaxial ilayer. Contrary to the $\mathrm{VGF}$ and $\mathrm{VCz}$ grown undoped $\mathrm{GaAs}$, the density of the circular arc dislocations was found to be small. Long screw dislocations were found in the $\mathrm{p}-\mathrm{i}$ interface with their starting points at the threading dislocations.
Fig. $7004, \overline{1} 17$ and $1 \overline{1} 7$ back reflection topographs of $\mathrm{p}$-side of sample D87

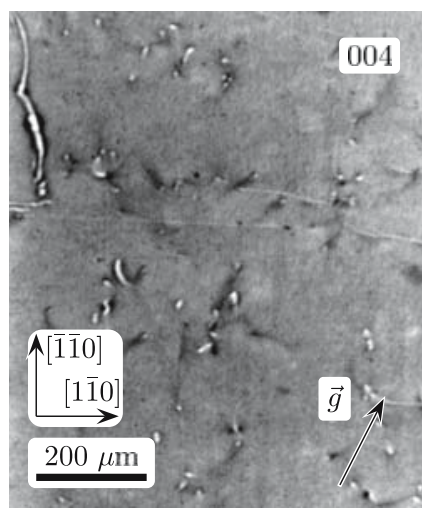

(a)

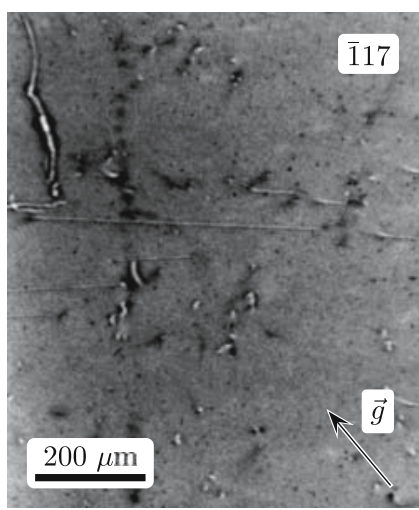

(b)

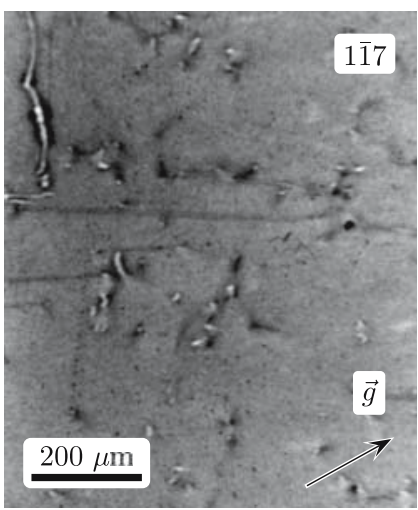

(c) 
The results of this work suggest that using undoped GaAs as a substrate rather than $\mathrm{n}^{+}$-type wafers, the creation of the misfit dislocation network at the interface between the intrinsic epilayer and the substrate could be avoided. Consequently, the density of the threading dislocations could be decreased. The topographs also show that it is possible to grow a misfit dislocation free n-layer onto the substrate side of a thick HVPE grown layer after a successful substrate removal process.

Acknowledgements This work was supported by the European Community - Research Infrastructure Action under the FP6 "Structuring the European Research Area" Programme (through the Integrated Infrastructure Initiative "Integrating Activity on Synchrotron and Free Electron Laser Science"). Also, we would like to thank Dr. C. Paulmann for his assistance at HASYLAB beamline F1 Topography.

\section{References}

1. M.J. Cardwell, J. Crystal Growth 70, 97 (1984)

2. C. Angulo Barrios, E. Rodriguez Messmer, M. Holmgren, A. Risberg, J. Halonen, S. Lourdudoss, J. Electron. Mater. 30, 987991 (2001)
3. C. Lynch, D. Bliss, T. Zens, D. Weyburne, J. Jimenez, M. Avella, P. S. Kuo, X. Yu, Characterization of HVPEgrown thick GaAs structures for IR and $\mathrm{THz}$ generation. Proceedings of the International Conference on Indium Phosphide and Related Materials Conference, 2006, 151-154

4. M.K. Kelly, R.P. Vaudo, V.M. Phanse, L. Görgens, O. Ambacher, M. Stutzmann, Jpn. J. Appl. Phys. 38, L217-L219 (1999)

5. A. Yasan, R. Mcclintock, K. Mayes, S.R. Darvish, P. Kung, M. Razeghi, R.J. Molnar, Opto-Electron. Rev. 10, 287-289 (2002)

6. T.O. Tuomi, J. Synchrotron Rad. 9, 174-178 (2002)

7. T. O. Tuomi, M. Juvonen, R. Rantamäki, K. Hjelt, M. Bavdaz, S. Nenonen, M.-A. Gagliardi, P. J. McNally, A. N. Danilewsky, E. Prieur, M. Taskinen, M. Tuominen,Mat. Res. Soc. Symp. Proc. 487, 459-464 (1998)

8. T.O. Tuomi, L. Knuuttila, J. Riikonen, P.J. McNally, W. Chen, J. Kanatharana, M. Neubert, P. Rudolph, J. Crystal Growth 237239, 350-355 (2002)

9. R.W.G. Wyckoff, Crystal Structures, 3rd edn. (Interscience Publishers, New York, 1948)

10. C. Domke, Ph. Ebert, M. Heinrich, K. Urban, Phys. Rev. B 54, 10288-10291 (1996)

11. B.D. Cullity, S.R. Stock, Elements of X-ray diffraction (Prentice Hall, New Jersey, 2001)

12. R. People, J.C. Bean, Appl. Phys. Lett. 47, 322-324 (1985)

13. L.H. Kuo, L. Salamanca-Riba, B.J. Wu, G.M. Haugen, J.M. DeBuydt, G. Hofler, H. Cheng, J. Vac.Sci. Technol. B 13, 16941704 (1995) 